\title{
Non-communicating Rudimentary Uterine Horn Pregnancy
}

\author{
Upadhyaya I. \\ Paropakar Maternity and Women's Hospital, Thapathali, Kathmandu, Nepal
}

\section{ABSTRACT}

Pregnancy in a non-communicating rudimentary horn is an extremely rare form of ectopic gestation. The rudimentary horn may or may not communicate with the uterine cavity with the majority of cases being non-communicating. The patient exhibits features of acute abdomen and carries a high risk of maternal death. Even modern scans remain elusive whereas laparatomy remains the confirmatory procedure for the diagnosis. Because of the varied muscular constitution in the thickness and distensibility of the wall of the rudimentary horn, pregnancy is accommodated for a variable period of gestation. Here, we report three cases of pregnancy in a non-communicating rudimentary horn of the uterus in different periods of gestation, their outcome and a review of the available literature.

Keywords: Mullerian anomalies, non-communicating rudimentary horn pregnancy, surgical management

\section{INTRODUCTION}

A non-communicating rudimentary horn is a rare type of Mullerian duct malformation. This results from defective fusion or defective absorption during embryonic life. The first case of a rudimentary horn was described by Manricean in 1669.1,2 A rudimentary horn pregnancy occurs in approximately $1: 100,000$ to $1: 140,000$ pregnancies. The possible explanation for pregnancy to occur is by transperitoneal migration of ovum through the abdominal cavity. This suggestion is based on the findings of corpus luteum in the contralateral ovary in 10 $\%$ of cases. ${ }^{3}$ Despite advanced ultrasound technology, antenatal diagnosis remains elusive with confirmatory diagnosis being made usually at laparotomy. The natural history of rudimentary horn pregnancy (RHP) is usually rupture of the pregnant horn during the second or third trimester resulting in a life-threatening hemoperitoneum. A study shows the incidence of such Mullerian anomaly to be higher than previously thought (i.e., $1 / 250$ women). ${ }^{4}$ Various subtypes of unicornuate uterus present $5 \%$ of all the congenital anomalies and are one of the least common malformations. More frequently, they do have a rudimentary horn (74 \%) which do not communicate to the contralateral uterus (72 \%). These non-communicating rudimentary horns are without endometrial cavity $(45 \%)$ and do not have a cervix or a vaginal outlet in some cases. ${ }^{2,5}$ Pregnancy is accommodated for a variable period of gestation because of the varied muscular constitution

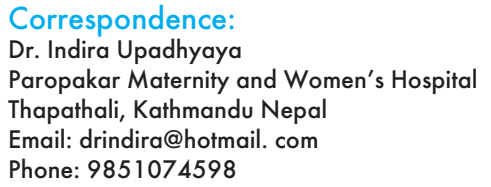

Correspondence:

Dr. Indira Upadhyaya

Paropakar Maternity and Women's Hospital

Thapathali, Kathmandu Nepal

Email: drindira@hotmail.com

Phone: 9851074598 
in the thickness and distensibility of the wall of the rudimentary horn. We here present three cases of non-communicating rudimentary horn with viable outcomes. Excision of the rudimentary horn was done at laparotomy in all three cases where the diagnosis was missed by ultrasonography.

\section{CASE REPORT}

\section{CASE 1}

A 23-year-old, $\mathrm{G}_{2} \mathrm{P}_{1}$ lady was admitted to this hospital with pain in the abdomen for one week and spotting per vaginum for one day. A week previously, because of sudden acute pain in the abdomen she had attended local clinic where the pain has been cured. After a urine test for pregnancy and by manual examination, it was concluded that she was carrying a four-month pregnancy. As she was on injection Depoprovera since three years, she was unaware of her pregnancy and so she was advised for a ultrasound scan. Trans-abdominal ultrasound scan revealed a 14-week intra-uterine fetal death with an early, low-lying placenta. Regarding her past history, sha had had a cesarean section for a healthy male child four years back.

On examination, her vitals were stable and the uterus was palpable, 14 - 16 weeks' size. On speculum examination, the cervix and vagina were healthy. The investigation showed, a slightly low hemoglobin of 10 gm \% while other laboratory parameters were within normal limits. Four doses of Misoprostol $100 \mathrm{mcg}$ in the posterior fornix given 12 hourly failed to expel the fetus. Two days later, Misoprostol $400 \mathrm{mcg}$ was kept in the posterior fornix 4 hours prior to evacuation. Perforation took place during the attempt of dilatation and curettage. Emergency laparotomy was done and revealed a pregnancy in the rudimentary horn of size $14 \times 14 \mathrm{~cm}$. The horn appeared non-communicating with the uterine cavity containing the product of conception. The uterus was normal in size with a small perforation of $0.5 \times 1 \mathrm{~cm}$ at its cornual end. The rudimentary horn with the right tube and the right ovary was excised. Complete haemostasis was achieved and the abdomen was closed in layers. The cut section of the horn showed a male macerated fetus of about 16 weeks gestation with the cord and the placenta. The postoperative period was uneventful and the patient was discharged a week later.

\section{CASE 2}

A 21-year-old primigravida attended the emergency room with complaints of pain abdomen with increasing severity for one day and per vaginal bleeding for 2 hours. She had been married for 6 months, had never used any contraceptives and gave a history of amenorrhea of 8 weeks. There was no history of giddiness or fainting episode or abdominal trauma. Her pregnancy test was positive.

On examination, she was afebrile, looked ill, pale, with a pulse of 120 beats/minute and a blood pressure of $100 / 70 \mathrm{~mm}$ of $\mathrm{Hg}$. The abdomen was soft with marked tenderness in the right iliac fossa without rebound tenderness. Per vaginal examination revealed the uterus to be of around 6 weeks size with the os closed. There was a tender cystic mass the size of which was product of conception of $10-12$ weeks in the right adnexae with the uterus deviated to the right side and bleeding was seen at the time of the examination. A provisional diagnosis of threatened abortion with tubo-ovarian mass was made. Lab parameters were within normal limits, except for the haemoglobin of $10.8 \mathrm{gm} / \mathrm{dL}$. And ultrasound of the abdomen showed a single dead fetus in the abdomen and it was not clearly mentioned in the report whether the pregnancy was intrauterine. Emergency laparotomy revealed an rudimentary horn pregnancy (RHP) of about 8 weeks gestation. Excision of the rudimentary horn along with the tube and the ovary was done. The postoperative period was uneventful (Figure 1).

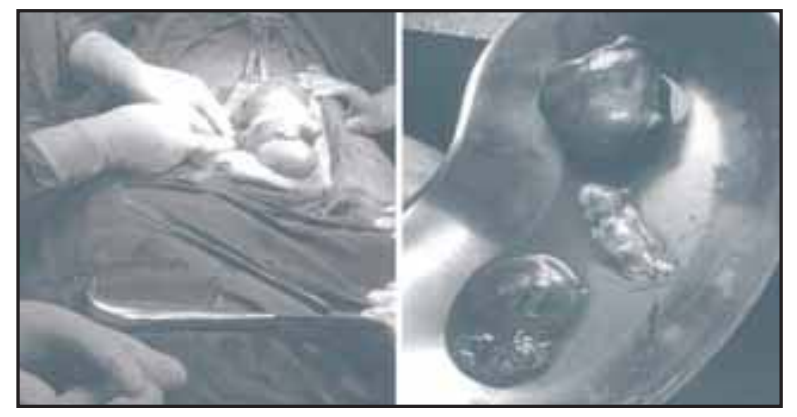

(a)

(b)

Figure 1. Case 2. (a) Before excision, pregnancy in a non-communicating rudimentary horn; (b) Excised rudimentary horn, with the fallopian tube, fetus and placenta.

\section{CASE 3}

A 30-year-old woman was admitted in labour after 39 weeks of pregnancy with pregnancy induced hypertension. Documented regular antenatal ultrasonograms at three, five and nine months were reported as normal intrauterine pregnancy. She had been married for 10 years with a history of sub-fertility treatment. A trial of vaginal delivery was stopped as she developed haematuria and a blood pressure of 160/110 $\mathrm{mm}$ of $\mathrm{Hg}$. An emergency caesarian section was done, the diagnosis of a non-communicating rudimentary horn 
pregnancy was made with a live female baby of a good apgar score and a weight of $2.6 \mathrm{~kg}$. A rudimentary horn excision and an ipsilateral salpingoophrectomy were performed. She was discharged on the seventh postpartum day. (Figure 2)

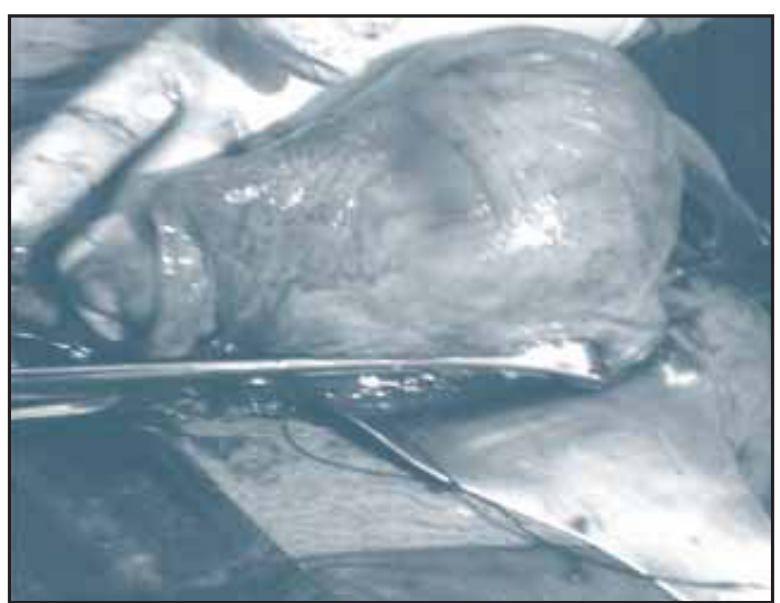

Figure 2.Case 3.Excised rudimentary horn, tube and ovary.

\section{DISCUSSION}

Pregnancy in a non-communicating rudimentary horn represents a form of ectopic gestation. The severity of uterine malformation relates inversely to the degree of the fusion defect of the primordial ducts. The majority of rudimentary horns are non-communicating to the main uterine cavity as reported in these three cases. Nahum et al showed that intraperitoneal sperm and ovum transmigration occur respectively in $50 \%$ and $40 \%$ of all cases of human pregnancy. Pregnancy in a rudimentary horn has been reported to be between $1: 100000$ to $1: 140000$ pregnancies. ${ }^{3}$ The review of the relevant literature in the 20th century by Nahum had 588 such cases. Two pregnancies of that series were rudimentary horn pregnancies. One was a twin pregnancy at 28-week gestation while the other pregnancy was of 37 weeks. Both presented with acute abdomen, underwent cesarean section and had live babies. During laparotomy, the patient was put in the lithotomy position. Per vaginum examination revealed a single cervix with an intact cervical canal. No communication with the normal uterine cavity was found. The cervical dilator placed in the cervical canal showed that the cervix was in continuity with the right horn and that the left horn was non-communicating with cervical canal. The babies were taken home with the mother like in the third case of our current study. In most cases of pregnancy in the rudimentary horn, the pregnancy lasts longer than in tubal pregnancy because of the variable musculature constitution in the thickness and distensibility of the wall of the rudimentary horn. The literature reports that $80-90 \%$ of rudimentary horn pregnancies rupture by the second trimester and $10 \%$ reach till term with a $2 \%$ fetal salvage rate ${ }^{1}$, where maternal mortality was $5.1 \% .^{1,2}$

It is also seen that some of the RHP cases are diagnosed only after an attempt to evacuate the uterus for termination of incorrectly-diagnosed intrauterine pregnancy, like in our first case. Though uterine anomaly can be diagnosed in only $14 \%$ of symptomatic patients by USG, in a review of 266 RHP, sensitivity of USG as a diagnostic tool was shown to be $26 \%$. Thus, the early diagnosis of RHP remains challenging. Noncommunicating rudimentary horn should be suspected whenever difficulty is encountered during termination of pregnancy as it is easy to miss this condition both clinically and by USG. ${ }^{4,5}$ Communicating rudimentary horns are less likely to be symptomatic before and during early pregnancy. The pain associated with rudimentary horns in pregnancy commences from the end of the first and beginning of the second trimester, like in our first and second cases. Vaginal bleeding is uncommon unless pregnancy is in the communicating horn. In our cases, one had spotting and another bleeding per vaginum. Difficulty in diagnosis during early pregnancy is quite common as there are no definite signs to distinguish this abnormal implantation from normal intra-uterine pregnancy, especially if it is anterior to the normal horn. Accurate diagnosis is possible only after laparotomy for acute abdomen. Tsafri et al suggested the following sonographic criteria for early diagnosis of RHP: a pseudo-pattern of an asymmetrical bi-cornuate uterus, absent visual continuity between the cervical canal and the lumen of the pregnant horn, and the presence of myometrial tissue surrounding the gestational sac. ${ }^{2}$ These criteria can help differentiate suspected RHP from cornual, intrauterine and pregnancy in a bicorunate uterus. Magnetic resonance imaging may have a major contribution to the diagnostic evaluation when pregnancy in a rudimentary horn is suspected. It offers multiplanar images without the hazards of ionizing radiation, is non-invasive and is able to show both the internal and external uterine structure. ${ }^{1,2}$ It has been reported that a patient had four previous laparotomies in which the correct diagnosis of the Mullerian anomaly was missed ${ }^{2}$ and had non- communicating rudimentary horn pregnancy. This is similar to our third case, who had had a previous cesarean that did not exclude the possibility of a rudimentary horn pregnancy.

The traditional management of RHP is laparotomy and surgical removal of the pregnant horn to prevent rupture and recurrennce. The fallopian tube on the side of the rudimentary horn must be removed in order to avoid 
Upadhyaya. Non Communicating Rudimentary Uterine Horn Pregnancy

tubal pregnancies. ${ }^{5,6-9}$ There are now other modern techniques for the management of rudimentary horn pregnancy like laparoscopic medical treatment. Surgery requires adequate equipment and experienced surgeons as the operation involves the risk of damage to the ureters mainly if endometriotic lesions and/or complete uretric duplication are present. So, during laparoscopy, it is mandatory to identify, and, if necessary, to perform ureterolysis prior to the resection of the uterine horn. That is why preoperative intravenous pyelography must be carried out systematically to reveal any urinary malformations which are associated with $30-40 \%$ of cases. ${ }^{9}$ Dicker et al reported the case of women who benefited from laparoscopic surgery of a rudimentary horn. ${ }^{6}$ Laparoscopic management of a uterine horn pregnancy is the most accurate diagnostic tool that allows efficient surgical management thereby avoiding laparotomy. ${ }^{10,11}$ The literatures report a successful medical abortion of a pregnancy within a noncommunicating rudimentary uterine horn. The use of methotrexate as reported by Samuels provides another treatment option and it can be a useful adjunct to surgical intervention. ${ }^{12}$

In conclusion, non-communicating rudimentary uterine horn pregnancy is a rare entity associated with lifethreatening consequences. Early diagnosis and early interventions will avoid maternal morbidity and mortality. These patients are advised to be screened for urinary tract anomalies. A complete USG examination should be performed on the aspect of the pregnancy and the pelvic anatomy. If USG remains inconclusive, the use of magnetic resonance imaging is suggested.

\section{REFERENCES}

1. Elsayegh A, Nwosu EC. Rupture of pregnancy in the communicating rudimentary uterine horn at 34 weeks. Hum. Reprod.1998;13(12):3566-68.

2. Tsafrir A, Rojansky N, Sela HY, Gomori JM, Nadjari M. Rudimentry horn pregnancy. Frist-trimester prerupture sonographic diagnosis and confirmation by magnetic resonance imaging. J Ultrasound Med 2005;24:219-23.

3. Nahum GG. Rudimentary uterine horn pregnancy. The $20^{\text {th }}$ century world wide experience of 588 cases. J Reprod. Med 2002;47(2):151-63.

4. Chopra S, Suri V, Agrawal N. Rudimentey horn pregnancy: Prerupture diagnosis and management. 2007;61(1):28-29.

5. Dasari P. Pre-rupture ultrasound diagnosis of rudimentary horn pregnancy. Biomed research 2007;18(3):185-87.

6. Dicker D, Nitke S, Shoenfeld A, Fish B, Meizner I, BenRafael Z. Laproscopic management of rudimentary horn pregnancy. Hum.Reprod.1998;13(9):2643-44.
7. Oral B, Guney M, Sonal S. Placenta accreta associated with a ruptured pregnant rudimentary uterine horn. Case report and review of the literature. Arch Gynecol Obstet. 2001; 265(2):100-2.

8. Nishi H, Funayama H, Fukumine N, Yagishita M, Nohira $\mathrm{T}$, Sujuki $\mathrm{Y}$ et al. Rupture of pregnant noncommunicating rudimentary horn with fetal salvage. A case report. Arch Gynecol Obstet. 2003;268(3):224-6.

9. Suguna RK, Vidya AT, Bushra K, Fathima. Rupture of non communicating rudimantary horn of uterus. AL Ameen J Med Sci.2009;2(1):85-86.

10. Arsian T, Bilgic E, Sentork MB, Yucel N. Rudimentary uterine horn pregnancy. A mystery diagnosis. Fertil Steril.2009; 92(6):2037.e1-3. Epub 2009 Oct 1.

11. Koul I, Wakhloo A, Gupta SB. Catastrophic haemorrhage after the rupture of rudimentary horn pregnancy. JK Practittoner 2006;13(4)212-13.

12. Edlman AB, Jensen JT, Lee DM, Nichols MD. Successful medical abortion of a pregnancy within a noncommunicating rudimentary uterine horn. Am J Obstet Gynecol.2003;189(3):886-7. 